\title{
High Vibration Sensors: Modelling, Design and Integration
}

\author{
Véronique Rochus ${ }^{1}$, Stefanie Gutschmidt ${ }^{1}$, Jean-Claude Golinval ${ }^{1}$, \\ Michel Saint-Mard ${ }^{3}$, Bruno Heusdens ${ }^{2,4}$, Fabrice Haudry ${ }^{2,4}$, Jacques Destiné ${ }^{4}$ \\ ${ }^{1}$ Aerospace \& Mechanical Engineering, department of the University of Liege, \\ Chemin des chevreuils, 14000 Liège, Belgium \\ V.Rochus@ulg.ac.be \\ ${ }^{2}$ MICROSYS laboratory (University of Liege, Belgium) \\ ${ }^{3}$ TAIPRO Engineering SA. \\ ${ }^{4}$ EMMI department of the University of Liege, Belgium
}

\begin{abstract}
Since many years, the University of Liège is involved in micro-electronics and microelectromechanical systems modelling, design and integration. Recently, the University of Liege had received the opportunity to build a brand new infrastructure (clean rooms - ISO 7) with specific equipments for packaging and MEMS characterisation. This new facility (clean rooms and equipments) enables the University to be very well positioned in the nano/micro-electronics modelling, analysis and packaging world and is now able to answer specific research and related industrial needs.

In this paper we consider the design of a vibration sensor in its significantly vibrating surroundings and investigate in its dynamical behaviour. Environmental vibrations affect the sensor part of the MEMS device and influence the choice of the "best" packaging methods for the application. Within the framework of packaging, we consider a simple test application ensuring best interconnection technology. Dynamical investigations include a preliminary analysis of the packaging and a separate finite-element analysis of the MEMS device (inside the package), testing the device under the condition of a harsh environment (high vibration spectral level). Computations are proposed in combination with experimental observations.
\end{abstract}

\section{MICROSYS - Packaging laboratory}

The aims of MICROSYS are to increase industrial awareness and help industrials to develop innovative prototypes of microsystems for all kinds of application niches.

The Microsys facility involves $200 \mathrm{~m}^{2}$ of clean rooms available for the interconnection and packaging activities for research and industrial prototyping in the field of microsystem. The interconnection and packaging of microsystems of course need the use of equipments and software of a high level technology. These tools allow us to design and assemble microsystems in agreement with a wide range of norms and harsh environment. All the available equipment is manual and versatile in order to allow the largest and innovative capabilities for packaging application.

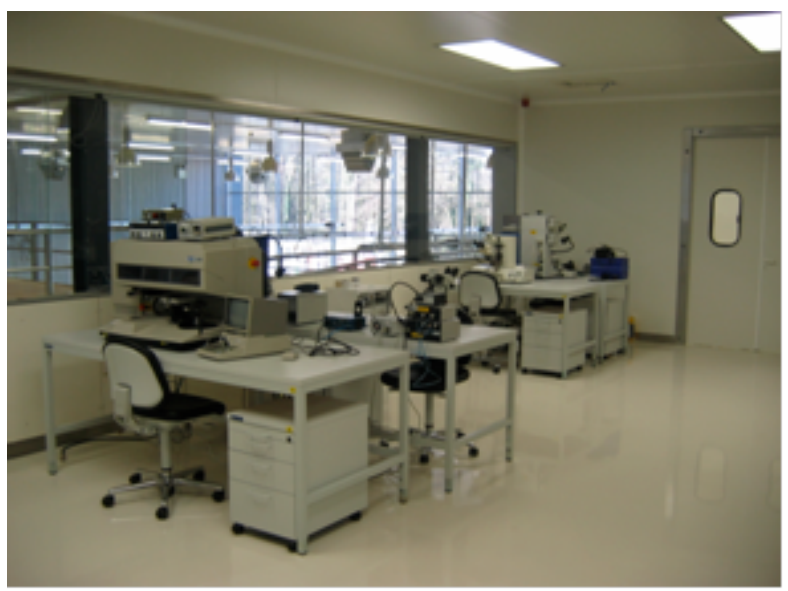

Figure 1: Clean room.

In summary, this facility allow to assemble, interconnect, encapsulate and characterize a wide range of microsystems, including wafer scribing (till 6"), plasma cleaning and activation, pick and place (die attach, flip chip), ultrasonic wire bonding (ball, wedge and bump), tests (wire pull, ball \& die shear test), microsystem encapsulation (potting, globe top, hermetic seam sealing), PCB gold plating, PCB machining and package decapsulation.

\section{MEMS laboratory}

The laboratory of "Structural Dynamics Research Group" has recently purchased new instruments including a scanning laser vibrometer MSA400 from Polytec and a vacuum cryogenic chamber from Janis. With this equipment we are able to investigate especially in the dynamical behaviour of MEMS, including experimental setups in vacuum until around 10-6 Torr for different temperatures ranging from $8 \mathrm{~K}$ to $450 \mathrm{~K}$. 


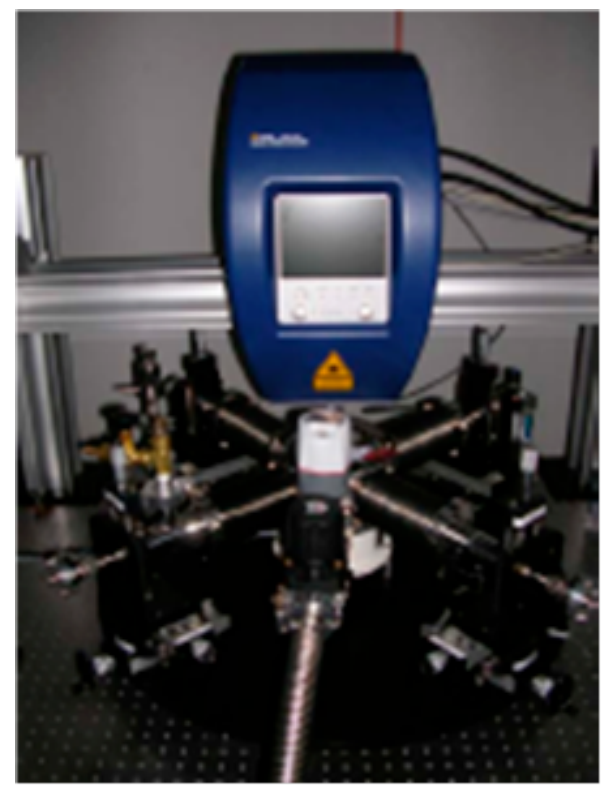

Figure 2 : Laser vibrometer MSA 400 Polytec.

\section{PACKAGING FOR NICHE APPLICATIONS}

Industrial applications induce several constraints on the choice and the assembly of Components Off The Shelf (COTS) (packaged and bare die). These constraints depend on the application and are often located in a harsh environment. In most of the cases, we need to integrate the microsystem in an existing system which then induces a lot of constraints like dimensions, power supply, and so on.

The choice of the right COTS is chosen after the following criteria:

\section{Functionality}

Functionnality is defined according to the component responding to the specifications. Most of the time, the first components to choose are the sensor and/or the actuator. Once choice is made, other electronic components are identifed according to the performane of correct measurements (frequency, threshold identification...), signal processing, power supply and communication with the "outside world".

\section{Resistance to environment}

The choice of COTS is also led by their ability to withstand the environmental constraints. Industrial applications are often located in a harsh environment i.e. high/low temperatures, vibrations, shocks, chemical resistance, high pressure, and so on.

In most of the cases, not all the components of a microsystem will withstand to all the environmental constraints. These components must be integrated in a dedicated "package". The role of this package is to protect the component against the environment and allow the requested measurement.

Each new design requires a new innovative solution regarding the packaging. Here are some specific examples:

- High temperature environment $\left(>125^{\circ} \mathrm{C}\right)$ : At these temperatures, the number of available components is drastically reduced. One way to protect conventional COTS is to design a cooling system e.g.heat sink, heat pipe, thermoelectric module, and so on.

- Environment with undesired vibrations: This has an impact on MEMS components and on the interconnection of any type of components. A good example is a MEMS in bare die form, interconnected with wire bonds. The wires are free to move laterally within a certain range (wire sweep). To avoid contact between wires and short circuits, we usually encapsulate them with an epoxy which also strengthen the bonds.

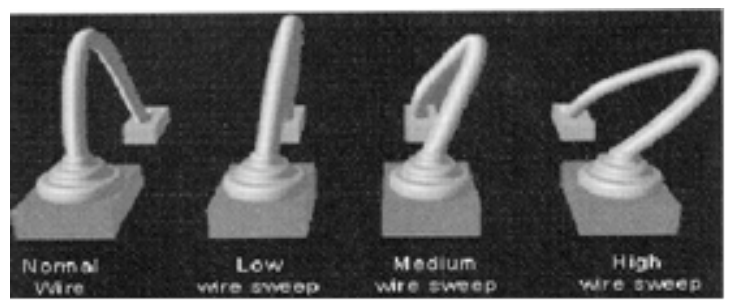

Figure 3a: Wire sweep.

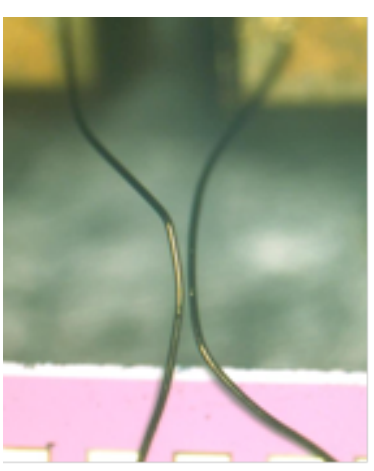

Figure 3b : Wire sweep.

In case of high vibration level, simulations show us the different vibration modes of a $25 \mu \mathrm{m}$ gold wire and an aluminum wire (see Figure 4). These simulations show also that the epoxy does not move in the same way as the die and wires, inducing stress on the bonds. An elegant and safe way to avoid unexpected wire displacement is to use the flip-chip process, with or without under fill. 
Table 1 shows that the first vibration mode could appear in high level vibration applications.
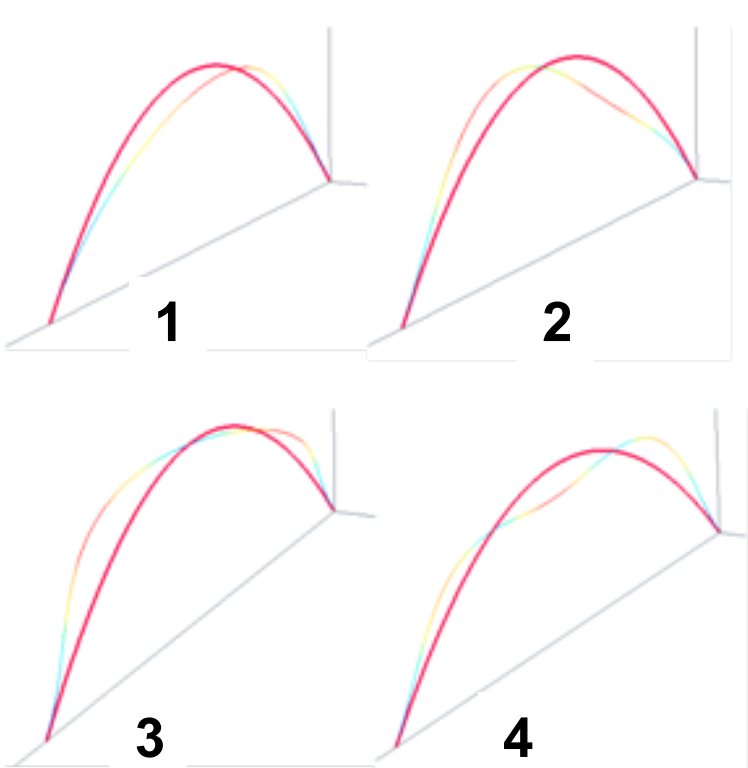

Figure 4 : Vibration modes of a $25 \mu \mathrm{m}$ wire.

Table 1 : Vibration modes of gold and aluminum wire.

\begin{tabular}{|c|c|c|}
\hline Modes & Gold & Alu \\
\hline 1 & $7.538 \mathrm{~Hz}$ & $18.992 \mathrm{~Hz}$ \\
\hline 2 & $20.832 \mathrm{~Hz}$ & $52.374 \mathrm{~Hz}$ \\
\hline 3 & $22.089 \mathrm{~Hz}$ & $55.621 \mathrm{~Hz}$ \\
\hline 4 & $43.170 \mathrm{~Hz}$ & $108.539 \mathrm{~Hz}$ \\
\hline
\end{tabular}

In this study we have also investigated the behavior of a bare die glued in a package. Simulations were made using a model of a ceramic package (see Figure 5) with a bare die glued with an adhesive (this is called "die attach"). Two configurations were simulated, one with the die encapsulated with an epoxy, the second without encapsulant.

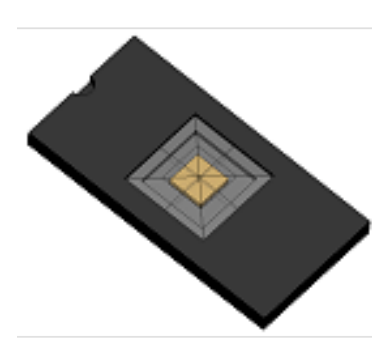

With encapsulant

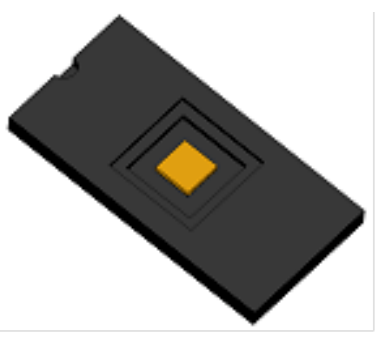

Without encapsulant
Figure 5 : Package model for simulation.

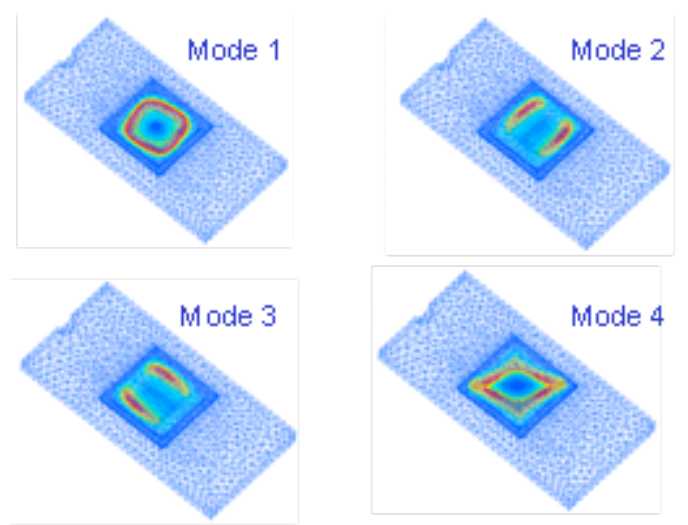

Figure 6 : Vibration modes of die attach with encapsulant.
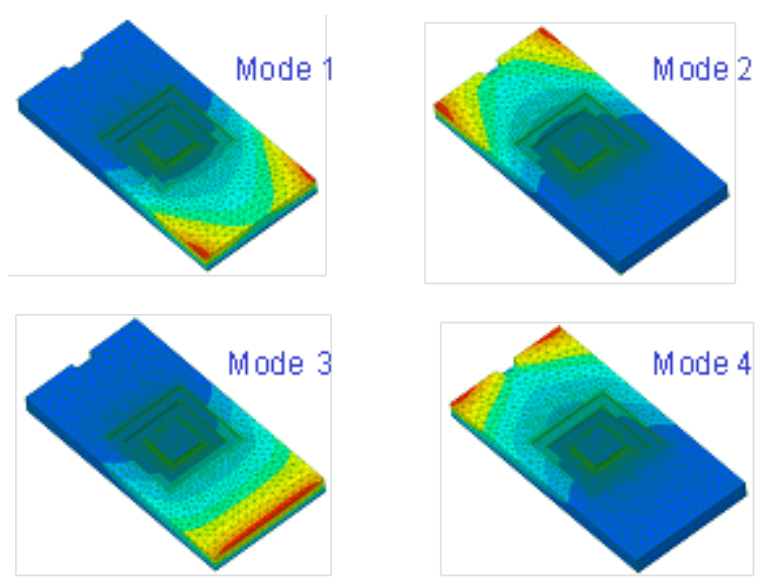

Figure 7 : Vibration modes of die attach without encapsulant.

Table 2 : Values of vibration modes of the die attach.

\begin{tabular}{|c|c|c|}
\hline & $\begin{array}{c}\text { With } \\
\text { encapsulant }\end{array}$ & $\begin{array}{c}\text { Without } \\
\text { encapsulant }\end{array}$ \\
\hline 1 & $444.434 \mathrm{~Hz}$ & $653.042 \mathrm{~Hz}$ \\
2 & $459.624 \mathrm{~Hz}$ & $654.061 \mathrm{~Hz}$ \\
3 & $459.891 \mathrm{~Hz}$ & $660.657 \mathrm{~Hz}$ \\
4 & $488.339 \mathrm{~Hz}$ & $665.584 \mathrm{~Hz}$ \\
5 & $506.977 \mathrm{~Hz}$ & $672.328 \mathrm{~Hz}$ \\
6 & $510.665 \mathrm{~Hz}$ & $674.231 \mathrm{~Hz}$ \\
7 & $510.807 \mathrm{~Hz}$ & $680.350 \mathrm{~Hz}$ \\
8 & $537.191 \mathrm{~Hz}$ & $684.584 \mathrm{~Hz}$ \\
9 & $548.213 \mathrm{~Hz}$ & $690.698 \mathrm{~Hz}$ \\
10 & $548.566 \mathrm{~Hz}$ & $694.607 \mathrm{~Hz}$ \\
\hline
\end{tabular}

In figure 6 , we can see that the encapsulant is moving, inducing stress on the die. Without encapsulant (see Figure 7), the vibration modes are located in the package, the die attach is not moving. Table 2 shows that the values of the different vibration modes are very high.

The different simulations of the MEMS and also the interconnections are specified later in this paper. 


\section{Costs}

Usually, people think that the components are the main factor of cost in a package. But in fact, the packaging represents 30 up to $75 \%$ of the overall cost of a microsystem. Awfully, we can say that 13/15 components projects failed because nobody had foreseen the packaging cost. For developing a new component "benchmark", any component designer must take into account the associated costs of the developming, packaging and assembling. That means, that, in most cases, it is not the best choice to take the cheapest component.

\section{Power consumption}

For autonomous microsystems, the power consumption is another important constraints and become a challenge when using an associated energy harvesting system. This has an impact on the choice of the components, but not on the package. An example is given in the next paragraph.

\section{Dimensions and weight}

In most of the cases, the microsystem must be integrated in an existing system with a fixed architecture. Dimensions of the microsystem are then fixed, which is an additional constraint for the design of the "package" and the choice of the components. In some applications (like aerospace), the weight must be as low as possible.

\section{DYNAMIC BEHAVIOUR OF MEMS IN HARSH ENVIRONMENT}

After considering the dynamic behaviour of the packaging, we will now focus on the effect of the external environment on the MEMS itself.

One of the research topics of the laboratory of "Structural Dynamics Research Group" is the study of static and dynamic behaviours of MEMS actuated by electrostatic forces. Main applications include e.g. micro-resonators for RF switches (radio-frequency switches) [6]. Such switches, also known by microbridges, consist of suspended micro-structures which upon applying a designed voltage carry out the wanted function. A potential difference appears between this device and the substrate and produces electrostatic forces attracting both electrodes together. In a first dynamical approach, the MEMS device is actuated electrostatically, i.e. no changes of conductivity in the material are included in the model. However, the applied voltage between the micro-structure (see Fig. 9) could consist of e.g. a bias and an oscillating voltage which in turn generates the dynamic response. In general, MEMS devices are subject to strongly non linear interactions $[4,5]$.
In order to study the dynamical behaviour of these structures numerically, we make use of 2D and 3D tools of a recently developed finite-element formulation for MEMS [2] in collaboration with the commercially available finite-element package Oofelie from Open Engineering. Dynamic responses of MEMS are measured with our recently purchased laboratory equipment. At this point, we would like to refer to another work of our group which is planned to be published in short. This paper will report on first experimental observations and investigations, and the evaluation of a reduced-order and finite-element model.

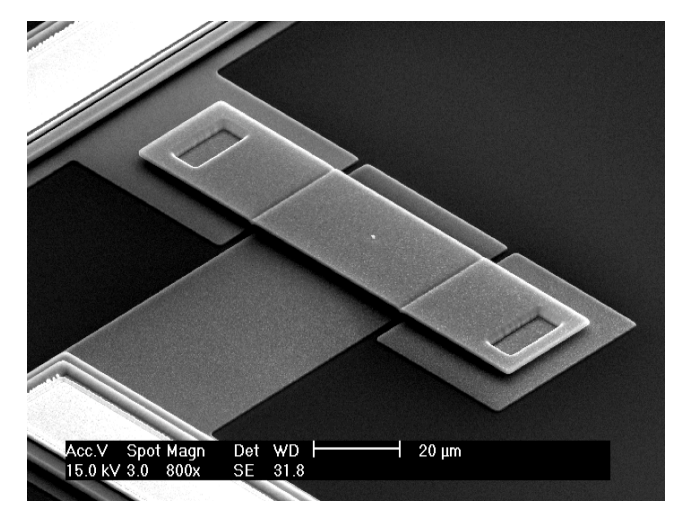

Figure 9 : Example of MEMS beam: Micro-bridge $300 \mu \mathrm{m} \times 30 \mu \mathrm{mx} 3 \mu \mathrm{m}$ (out of plane motion).

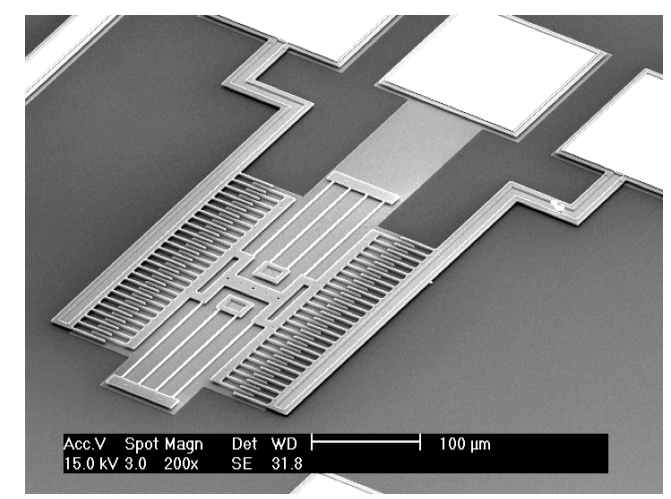

Figure 10 : Example of MEMS beam: Micro-resonator (in plane motion).

The goal of this research and collaboration with the MICROSYS laboratory is to study the behaviour of MEMS in relation to the aforementioned packaging challenges such as high external vibration.

External vibrations will have a significant effect on the dynamic behaviour of the structure. Also, it is well reported in literature that the dynamic instability of the device occurs before the static instability $[7,8,9]$, i.e. in terms of input voltages, the dynamic pull-in threshold occurs at lower voltages than the static pull-in point of the system. If such a pull-in happens uncontrolled or is 
unexpected for the choice of parameters the device, often times, is irreversibly damaged. Thus, simulations and experimental tests are performed to identify such instabilities precisely which eventually will lead into qualitative understanding to avoid or predict such behaviours.

The next section is divided into three parts. We will first explain the nonlinearity occurrence due to electrostatic forces using a one dimensional model. Then, a finite-element method is used to model the real micro-device. In the third part, simulations from the finite-element model will first address typical microdevice conditions before the simulations also include the external vibrations (representing the harsh environment).

\subsection{Lumped Mass Model of Electromechanical Model}

In order to understand the physical phenomena of electro-mechanical coupling, the reference problem shown in Fig. 12 is considered. It consists of a capacitor made of two parallel plates between which a voltage is applied. The upper plate is supported by a spring and the lower plate is grounded. This massspring model is representative of the mode of operation of electrostatically actuated MEMS devices. It serves the following explanations and is not meant for evaluation purposes of theoretical approaches in comparison to experimental observations

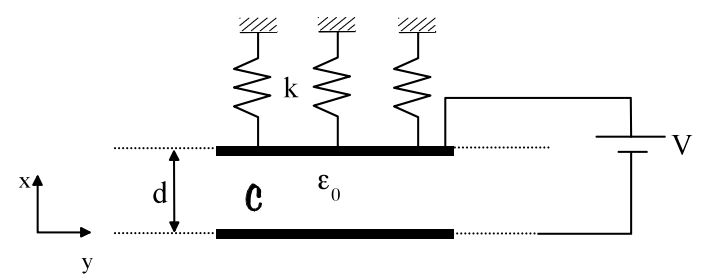

Figure 12. Lumped model of electromechanical coupling.

For the sake of simplicity, the electrodes of the capacitor are considered as infinite plates and the electric charges are supposed to be evenly distributed over the surfaces. This approximation allows to neglect fringing fields and to reduce the system to a onedimensional problem. The capacitor is also considered to be in vacuum and no damping, up to this point, is included in the model. The dynamic equilibrium equation of the system is:

$$
m \ddot{d}=-k\left(d-d_{0}\right)-\frac{1}{2} \varepsilon_{0} \frac{V^{2}}{d^{2}}
$$

where $d$ is the distance between the two plates; $m$ is the mass of the upper electrode; $k$ is the spring stiffness and $\varepsilon_{0}$ is the permittivity of free space. Note, that the dynamic behaviour of the structure depends on the applied voltage $V$ and on the initial gap $d_{0}$ between the plates.

The dynamic behaviour of the coupled system corresponding to different initial conditions are shown in the phase diagrams of Figure 13 (assuming $\left.\omega_{0}{ }^{2}=k / m=1\right)$. We observe that there is a stable zone in which the system oscillates around an equilibrium. However, for different initial conditions such as outside the separatrix, the electrodes snap into contact.

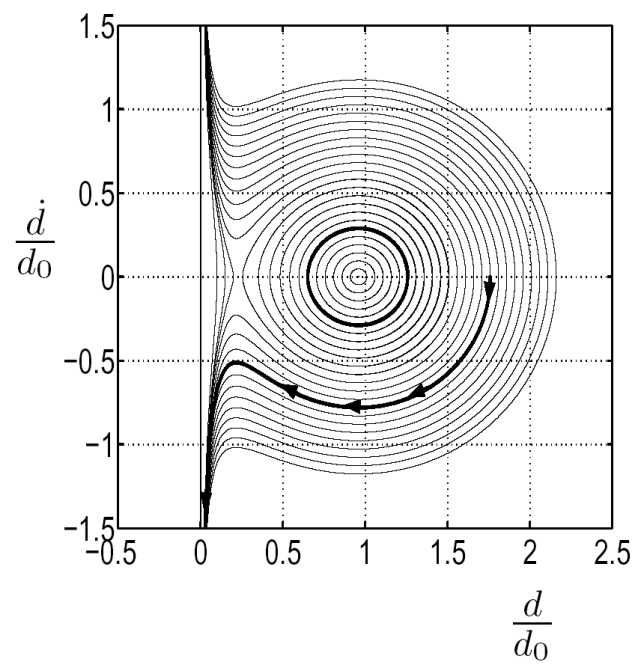

Figure 13 Phase diagram of the lumped model.

\subsection{Finite Element Model}

Considered is a micro-bridge of the length $300 \mu \mathrm{m}$, width $30 \mu \mathrm{m}$ and thickness $3 \mu \mathrm{m}$, see Figures 9 and 14 . The initial gap between the structure and the lower electrode is $3 \mu \mathrm{m}$. The static pull-in voltage is estimated at $18 \mathrm{~V}$.

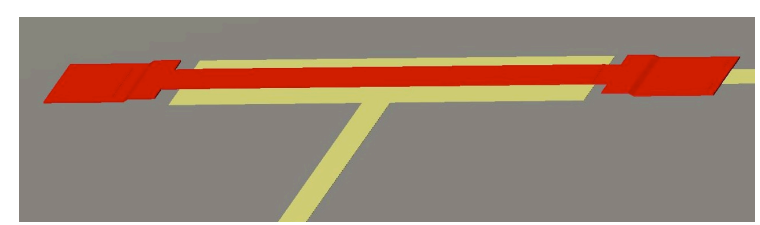

Figure 14 MEMS Model.

An oscillating voltage of $5 \mathrm{~V}$ is applied to excite the structure at resonance $(184 \mathrm{kHz})$. Computations, depicted in Figures 15, 16, and 17, 18, have been performed for a relatively low damping. We observe, by looking at the phase diagram and the time response (see Figures 15 and 16), that the structure for an AC input voltage of $5 \mathrm{~V}$ oscillates around an equilibrium position and converges to a stable limit cycle. 


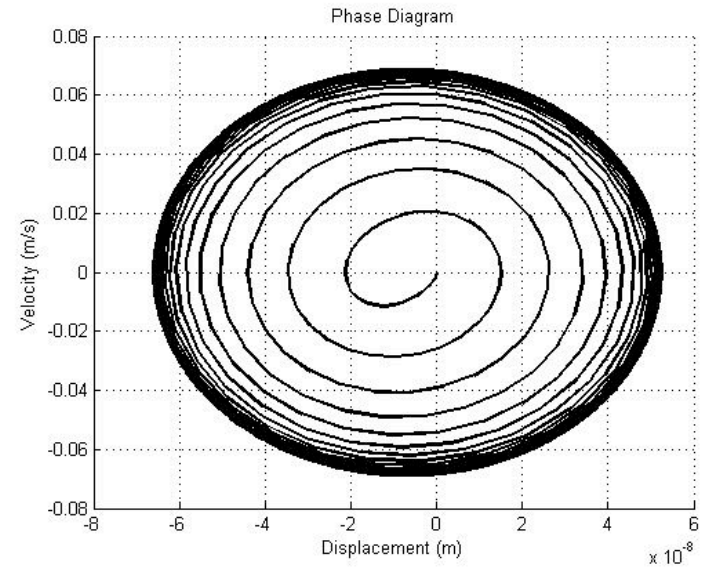

Figure 15: Phase diagram for 5V AC.

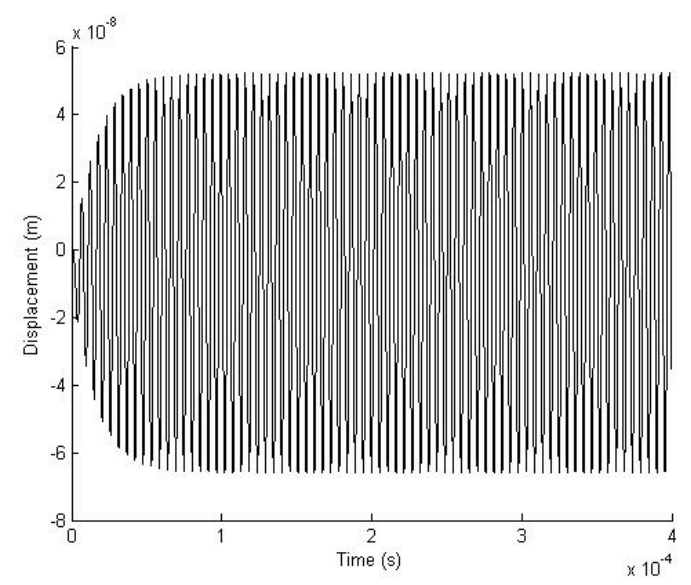

Figure 16: Time response for $5 \mathrm{~V}$ AC.

The same result is evident if $10 \mathrm{~V}$ are applied as the input voltage (Figures 17 and 18). The behaviour is found stable.

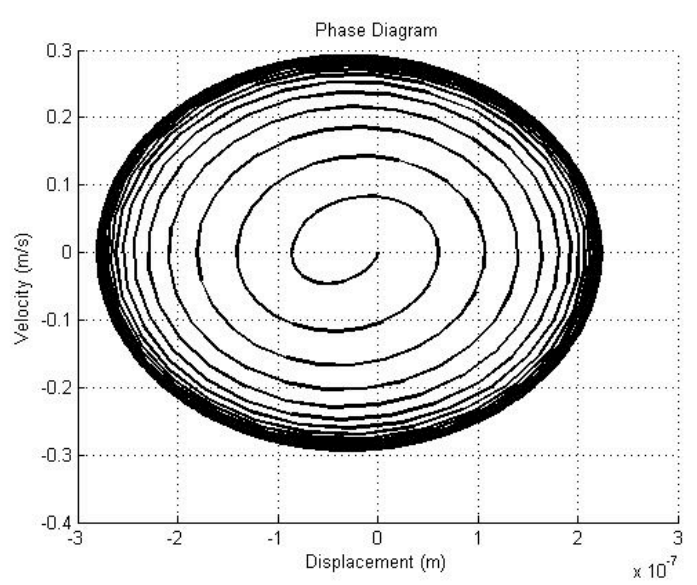

Figure 17: Phase diagram for 10V AC.

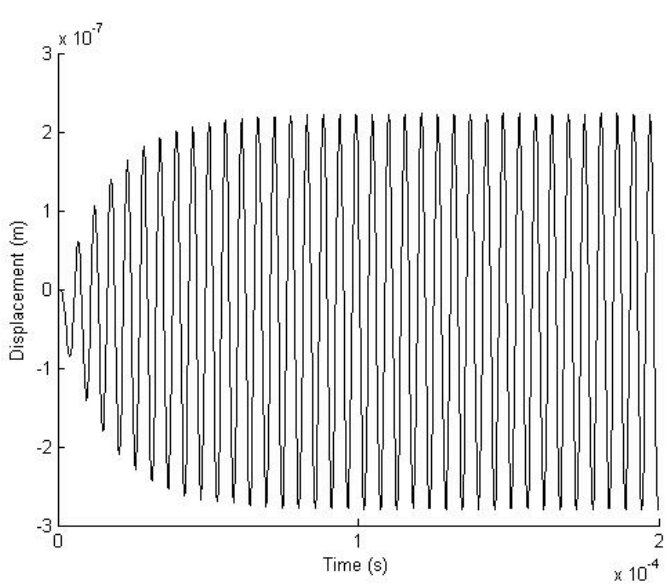

Figure 18: Time response for 10V AC.

In the following, the MEMS device, in addition to the previous dynamic actuation, will undergo external vibrations, simulating a "harsh environment". A representative set of parameters describing such a harsh environment would be e.g. an acceleration of $11 \mathrm{G}$ at around 2000Hz. In Figures 19 and 20, we observe, that for an input voltage of $5 \mathrm{~V}$ and the simulation of the harsh environment, the system remains stable.

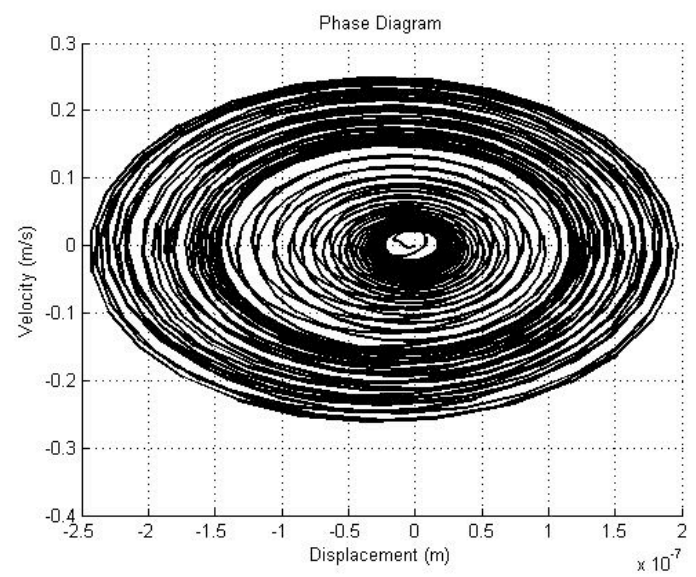

Figure 19: Phase diagram for $5 \mathrm{~V}$ and external vibrations of $11 \mathrm{G}$ at $2000 \mathrm{~Hz}$.

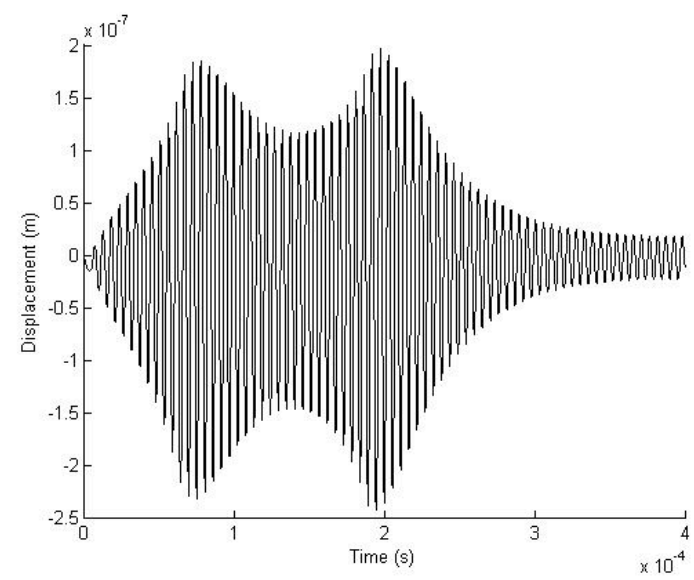

Figure 20: Time response for $5 \mathrm{~V}$ and external vibrations of $11 \mathrm{G}$ at $2000 \mathrm{~Hz}$. 
However, for the higher input voltage of $10 \mathrm{~V}$, the external vibrations add significantly to the energy input of the system, thus, resulting in an unstable behaviour of the MEMS device. The electrodes (micro-beam and bottom electrode) stick together as indirectly shown in Figures 21 and 22.

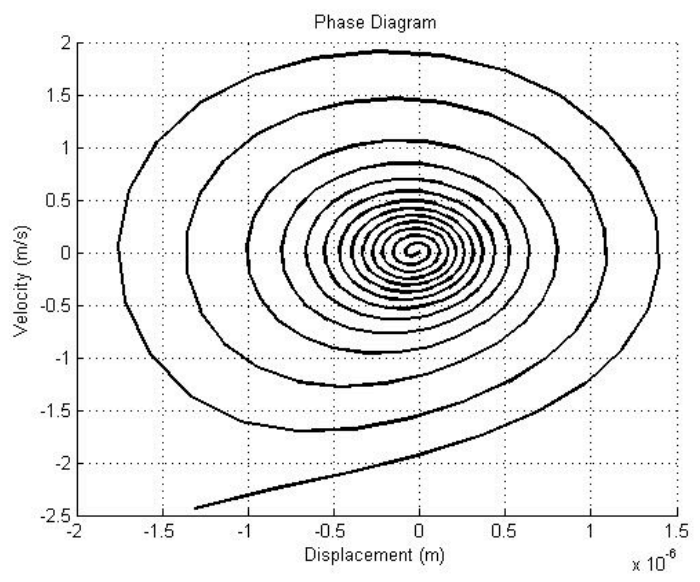

Figure 21: Phase diagram for $10 \mathrm{~V}$ and external vibrations of $11 \mathrm{G}$ at $2000 \mathrm{~Hz}$.

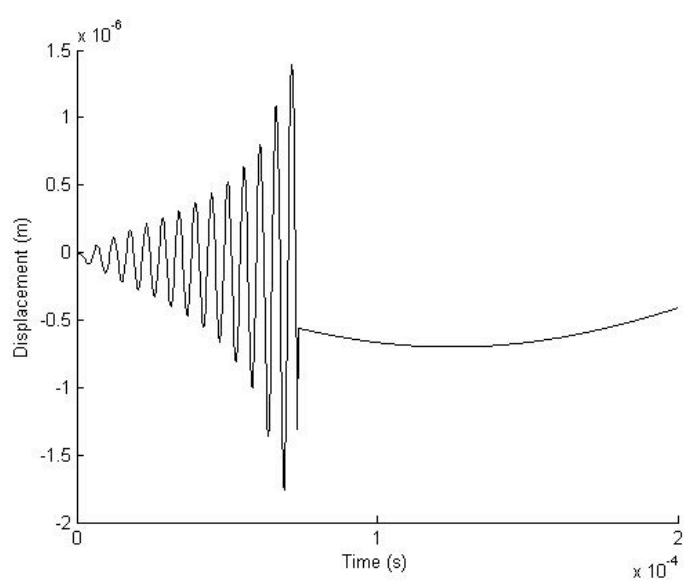

Figure 22: Time response for $10 \mathrm{~V}$ and external vibrations of $11 \mathrm{G}$ at $2000 \mathrm{~Hz}$.

\section{CONCLUSIONS}

For all kinds of research areas (whether for design of a new electronic component or integration of microelectronic in an industrial application) packaging as well as simulations are very important. In particular, we showed in this paper that when considering MEMS in a harsh environment, it is fundamental to take into account the interconnection and the packaging (into which the MEMS device is integrated). Thus, simulations must be done for the MEMS as well as for the whole micro-system to understand the influence of the package and interconnection on the MEMS. These simulations need to be evaluated by measurements on a real micro-system. We also showed that a lot of industrial processes or products need a specific packaging approach in order to achieve success in a final solution of a MEMS device made and kept functional in a safe packaging device.

As a general conclusion, most of the developed micro-systems are designed for harsh environments, hard to access location or maximization of the user comfort. Therefore, a particular effort is required in terms of design of such packaging systems.

\section{ACKNOWLEDGEMENT}

The first author acknowledges the financial support of the Belgian National Fund for Scientific Research.

The authors want to acknowledge all the supporting companies which are confident in our research activities. In particular, we want to acknowledge Techspace Aero, Magotteaux, CMI, Arcelor, Open Engineering for their special supports.

\section{REFERENCES}

1. Shankara K. Prasad, "Advanced Wirebond interconnection Technology", Kluwer Academic Publishers

2. Monolithic Modeling of Electro-mechanical Coupling in Micro-Structures, V. Rochus, D.J. Rixen, J.-C. Golinval, International Journal for Numerical Methods in Engineering, 2006, John Wiley \& Sons, Vol. 65/4, pp. 461-493

3. V.Rochus, D.J.Rixen, J.C. Golinval, "Electrostatic Coupling of MEMs Structures: Transient Simulations and Dynamic Pull-in." The 2004 Workshop on Coupled Problems, Processes, and Phenomena: Modelling, Control, and Analysis, Orlando, 2004.

4. Y. Bromberg, M.C. Cross, R. Lifshitz. Phys. Rev. E 73(016214), 1-8, 2006.

5. B. Ilic, Y. Yang, K. Aubin, R. Reichenbach, S. Krylov, H.G. Craighead. Nano Letters, 5(5):925-929, 2005.

6. C. Goldsmith, J. Randall, S. Eshelman, T.H. Lin, D. Denniston, S. Chen, and B. Norvell. IEEE

MTT-S International Microwave Symposium Digest, 2:1141-1144, 1996.

7. S. Krylov, R. Maimon. "Pull-in dynamics of an elastic beam actuated by continuously distributed electrostatic force," J. Vib. Acoust., 126(3), pp. 332$342,2004$. 
8. S. Krylov. "Lyapunov exponents as a criterion for the dynamic pull-in instability of electrostatically

actuated microstructures," Int. J. of Nonlin. Mech., 42, pp. 626-642, 2007.

9. M. Younis, E. Abdel-Rahman, A. Nayfeh. “A reduced-order model for electrically actuated microbeam-based mems," J. Microelectromech. Syst., 12(5), pp. 672-680, 2003

10. M. Saint-Mard et al, "Smart sensors for all over industrial application niches", Smart System Intergration conference, Brussels, March 2009 